International Journal of Pure and Applied Mathematics

Volume 116 No. 1 2017, 125-128

ISSN: 1311-8080 (printed version); ISSN: 1314-3395 (on-line version)

url: http://www.ijpam.eu

doi: $10.12732 /$ ijpam.v116i1.12

\title{
ON THE WEIGHTED COMPOSITION OPERATORS ON WEIGHTED BANACH SPACES
}

\author{
Bahmann Yousefi $^{1 \S}$, Fatemeh Zangeneh ${ }^{2}$ \\ ${ }^{1,2}$ Department of Mathematics \\ Payame Noor University \\ P.O. Box: 19395-3697, Tehran, IRAN
}

\begin{abstract}
In this paper we give conditions under which the numerical range of a weighted composition operator acting on $H^{p}(\beta)$ is closed.
\end{abstract}

AMS Subject Classification: 47B37, 47A16

Key Words: numerical range, weighted Hardy space, weighted composition operator

\section{Introduction}

Let $\{\beta(n)\}$ be a sequence of nonzero complex numbers with $\beta(0)=1$ and $1 \leqslant p<\infty$. We consider the space of sequences $f=\{\hat{f}(n)\}_{n=0}^{\infty}$ such that

$$
\|f\|^{p}=\|f\|_{\beta}^{p}=\sum_{n=0}^{\infty}|\hat{f}(n)|^{p}|\beta(n)|^{p}<\infty .
$$

The notation $f(z)=\sum_{n=0}^{\infty} \hat{f}(n) z^{n}$ will be used weather or not the series converges for any value of $z \in \mathbb{D}$. These are called formal power series. Let $H^{p}(\beta)$ denotes the space of such formal power series. It is called a weighted Hardy space. Let $f_{k}(z)=z^{k}$, then $\left\{f_{k}\right\}_{k}$ forms a basis for the reflexive Banach space $H^{p}(\beta)$. Note that the dual of $H^{p}(\beta)$ is $H^{q}\left(\beta^{p / q}\right)$, where $1<p<\infty, \frac{1}{p}+\frac{1}{q}=1$ and $\beta^{p / q}=\left\{\beta(n)^{p / q}\right\}_{n=0}^{\infty}$. If $\{\beta(n)\}_{n}$ is a sequence of positive numbers, with

Received: $\quad$ April 10, 2017

Revised: July 3, 2017

Published: August 29, 2017

${ }^{\S}$ Correspondence author (c) 2017 Academic Publications, Ltd. url: www.acadpubl.eu 
$\underline{\lim } \beta(n)^{\frac{1}{n}}=1$, then $H^{p}(\beta)$ is a Banach space of analytic functions on $\mathbb{D}$. The Hardy, Bergman and Dirichlet spaces can be viewed as we consider respectively that $\beta(n) \equiv 1, \beta(n)=(n+1)^{-1 / 2}$ and $\beta(n)=(n+1)^{-1 / 2}$.

Let $\lambda$ be a complex number, the functional of evaluation at $\lambda, e_{\lambda}$, is defined by $e_{\lambda}(p)=p(\lambda)$, for all polynomials $p$. Also, $\lambda$ is said to be a bounded point evaluation on $H^{p}(\beta)$ if the function $e_{\lambda}$ extends to be a bounded linear functional on $H^{p}(\beta)$. In this case we have $e_{\lambda}(f)=f(\lambda), f \in H^{p}(\beta)$.

Let $\varphi$ be an analytic self map of $\mathbb{D}$ and $\psi$ belongs to the set of multipliers of $H^{p}(\beta)$ that is denoted by $M\left(H^{p}(\beta)\right)$. A weighted composition operator $C_{\psi, \varphi}$ maps an analytic function $f \in H^{2}(\beta)$ into $C_{\psi, \varphi} f(z)=\psi(z) f(\varphi(z))$. The function $\varphi$ is called the composition map and the function $\psi$ is called the multiplier map. We will use the notations $H(\mathbb{D})$ and $C(\overline{\mathbb{D}})$ to denote the set of analytic functions on $\mathbb{D}$ and the set of continuous functions on $\overline{\mathbb{D}}$, the closure of $\mathbb{D}$.

In this paper we will give a definition for the numerical range of an operator acting on Banach spaces and then we will investigate it's closedness for weighted composition operators acting on the weighted Hardy spaces $H^{p}(\beta)$. For some sources on the topics of this paper one can refer to [1-7].

\section{Main Result}

In this section we investigate the closedness of the numerical range of composition operators acting on the sequence Banach spaces $H^{p}(\beta)$. from now on we consider that $H^{p}(\beta) \subset H(\mathbb{D})$.

Definition 2.1. Let $X$ be a reflexive Banach space and $T \in B(X)$. The numerical range of $\mathrm{T}$ is defined by

$$
W(T)=c o(V(T))
$$

where $\operatorname{co}(V(T))$ is the convex hull of $V(T)$ and

$$
V(T)=\left\{x^{*}(T(x)): x \in X, x^{*} \in X^{*} ;\|x\|=\left\|x^{*}\right\|=x^{*}(x)=1\right\} .
$$

Theorem 2.2. Let $\frac{1}{p}+\frac{1}{q}=1$ and $\left\|e_{\lambda}\right\| \rightarrow \infty$ as $\lambda \rightarrow z_{0}$ for some $z_{0} \in \partial \mathbb{D}$. Suppose $\varphi$ is any holomorphic self-map of the unit disk $\mathbb{D}$ such that $\sum_{n \geq 0} \frac{\varphi\left(z_{0}\right)^{n}}{z_{0}^{n} \beta(n)^{q}}$ is finite and let $\psi \in C(\bar{D}) \cap M\left(H^{p}(\beta)\right)$. If $C_{\psi, \varphi}$ is bounded on $H^{p}(\beta)$, then $0 \in \bar{W}\left(C_{\psi, \varphi}\right)$. 
Proof. Let $0 \neq \lambda \in \mathbb{D}$, and $\alpha_{\lambda}$ be such that $|\lambda|=\lambda \alpha_{\lambda}$. Note that

$$
e_{\lambda}(z)=\sum_{n=0}^{\infty} \frac{\bar{\lambda}^{n}}{\beta(n)^{p}} z^{n}
$$

and put

$$
f_{\lambda}(z)=\sum_{n=0}^{\infty} \frac{\alpha_{\lambda}^{n q} \lambda^{\frac{n q}{p}}}{\beta(n)^{q}} z^{n} .
$$

Then, $e_{\lambda} \in H^{p}(\beta)^{*}$ and $f_{\lambda} \in H^{p}(\beta)$. Indeed, $\left\|e_{\lambda}\right\|_{q}^{q}=\left\|f_{\lambda}\right\|_{p}^{p}$. Hence, $\left\|f_{\lambda}\right\|_{p}=$ $\left\|e_{\lambda}\right\|_{q}^{\frac{q}{p}}$ and so

$$
\left\|f_{\lambda}\right\|_{p}\left\|e_{\lambda}\right\|_{q}=\left\|e_{\lambda}\right\|_{q}^{q}
$$

Also,

$$
\begin{aligned}
<f_{\lambda}, e_{\lambda}> & =\sum_{n \geq 0} \frac{\alpha_{\lambda}^{n q} \lambda^{\frac{n q}{p}}}{\beta(n)^{q}} \lambda^{n}=\sum_{n \geq 0} \frac{\left(\alpha_{\lambda} \lambda\right)^{n q}}{\beta(n)^{q}} \\
& =\sum_{n \geq 0} \frac{|\lambda|^{n q}}{\beta(n)^{q}}=\left\|e_{\lambda}\right\|_{q}^{q} .
\end{aligned}
$$

So by $(*)$, we get

$$
<\frac{f_{\lambda}}{\left\|f_{\lambda}\right\|_{p}}, \frac{e_{\lambda}}{\left\|e_{\lambda}\right\|_{q}}>=1
$$

Put

$$
L_{\lambda}=<C_{\psi, \varphi}\left(\frac{f_{\lambda}}{\left\|f_{\lambda}\right\|_{p}}\right), \frac{e_{\lambda}}{\left\|e_{\lambda}\right\|_{q}}>
$$

Then $L_{\lambda} \in V\left(C_{\psi, \varphi}\right) \subseteq W\left(C_{\psi, \varphi}\right)$. But,

$$
\begin{aligned}
L_{\lambda} & =<\frac{f_{\lambda}}{\left\|f_{\lambda}\right\|_{p}}, C_{\psi, \varphi}^{*}\left(\frac{e_{\lambda}}{\left\|e_{\lambda}\right\|_{q}}\right)> \\
& =<\frac{f_{\lambda}}{\left\|f_{\lambda}\right\|_{p}}, \psi(\lambda) \frac{e_{\varphi(\lambda)}}{\left\|e_{\lambda}\right\|_{q}}> \\
& =\frac{\psi(\lambda)}{\left\|e_{\lambda}\right\|_{q}^{q}} f_{\lambda}(\varphi(\lambda)) \\
& =\frac{\psi(\lambda)}{\left\|e_{\lambda}\right\|_{q}^{q}} \sum_{n \geq 0} \frac{\alpha_{\lambda}^{n q} \lambda^{\frac{n q}{p}}}{\beta(n)^{q}} \varphi(\lambda)^{n} \\
& =\frac{\psi(\lambda)}{\left\|e_{\lambda}\right\|_{q}^{q}} \sum_{n \geq 0} \frac{|\lambda|^{n q}}{\lambda^{n} \beta(n)^{q}} \varphi(\lambda)^{n}
\end{aligned}
$$


So if $\lambda \rightarrow z_{0} \in \partial \mathbb{D}$, then we have

$$
\left\|e_{\lambda}\right\|_{q}^{q} L_{\lambda} \rightarrow \psi\left(z_{0}\right) \sum_{n \geq 0} \frac{\varphi\left(z_{0}\right)^{n}}{z_{0}^{n} \beta(n)^{q}} .
$$

Since $\sum_{n \geq 0} \frac{1}{\beta(n)^{q}}=\infty$, we get $L_{\lambda} \rightarrow 0$ as $\lambda \rightarrow z_{0}$. Hence,

$$
0 \in \bar{V}\left(C_{\psi, \varphi}\right) \subseteq \bar{W}\left(C_{\psi, \varphi}\right)
$$

and this completes the proof.

Theorem 2.3. Let $\frac{1}{p}+\frac{1}{q}=1$ and $\left\|e_{\lambda}\right\| \rightarrow \infty$ as $\lambda \rightarrow z_{0}$ for some $z_{0} \in \partial \mathbb{D}$. Suppose $\varphi$ is any holomorphic self-map of the unit disk $\mathbb{D}$ such that $\sum_{n \geq 0} \frac{\varphi\left(z_{0}\right)^{n}}{z_{0}^{n} \beta(n)^{q}}$ is finite and let $\psi \in C(\bar{D}) \cap M\left(H^{p}(\beta)\right)$. If $C_{\psi, \varphi}$ is compact, then $0 \in W\left(C_{\psi, \varphi}\right)$ if and only if $W\left(C_{\psi, \varphi}\right)$ is closed.

Proof. If $W\left(C_{\psi, \varphi}\right)$ is closed, then by Theorem 2.2, $0 \in W\left(C_{\psi, \varphi}\right)$. Let $\alpha \in \bar{W}\left(C_{\psi, \varphi}\right)$. This implies that there exists a constant $0 \leq c \leq 1$ such that $\alpha \in c W\left(C_{\psi, \varphi}\right)$. If $\alpha \neq 0$ and $c \neq 0$, then $\frac{\alpha}{c} \in W\left(C_{\varphi}\right)$. Since $W\left(C_{\psi, \varphi}\right)$ is convex and $0 \in W\left(C_{\psi, \varphi}\right)$, thus $\alpha \in W\left(C_{\psi, \varphi}\right)$. Therefore,

$$
\bar{W}\left(C_{\psi, \varphi}\right) \subseteq W\left(C_{\psi, \varphi}\right) \subseteq \bar{W}\left(C_{\psi, \varphi}\right) .
$$

This implies that $W\left(C_{\psi, \varphi}\right)$ is closed and so the proof is complete.

\section{References}

[1] F.F. Bonsall and J. Duncan, Numerical ranges II, London Math. Soc. Lecture Note Series 10, Cambridge, 1973.

[2] C. Cowen and B. MacCluer, Composition Operators on Spaces of Analytic Functions, CRC Press, 1995.

[3] K.E. Gustafson and D.K.M. Rao, Numerical Range, the Field of Values of Linear Operators and Matrices, Springer-Verlag, New York, 1997.

[4] B. Yousefi, On the space $l^{p}(\beta)$, Rend. Circ. Mat. Palermo, XLIX, No. 2 (2002), 115-120.

[5] B. Yousefi, Unicellurarity of the multiplication operator on Banach spaces of formal power series, Studia Mathematica, 147 (2001), 201-209.

[6] B. Yousefi and Y.N. Dehghan, Reflexivity on weighted Hardy spaces, Southeast Asian Bulletin of Mathematics, 28 (2004), 587-593.

[7] B. Yousefi, On the eighteenth question of Allen Shields, International Journal of Mathematics, 16, No. 1 (2005), 37-42. 